Review Articles

\title{
Pain and Suffering in Invertebrates: An Insight on Cephalopods
}

\author{
${ }^{1}$ Giorgia della Rocca, ${ }^{1}$ Alessandra Di Salvo, ${ }^{1}$ Giacomo Giannettoni and ${ }^{2}$ Mary Ellen Goldberg \\ ${ }^{\mathbf{1} D i p a r t i m e n t o ~ d i ~ M e d i c i n a ~ V e t e r i n a r i a, ~ U n i v e r s i t a ̀ ~ d e g l i ~ S t u d i ~ d i ~ P e r u g i a, ~ I t a l y ~}$ \\ ${ }^{2}$ Executive Secretary International Veterinary Academy of Pain Management, \\ Instructor VetMedTeam, LLC, Boynton Beach, FL, USA
}

Article history

Received: 31-12-2014

Revised: 2-03-2015

Accepted: 9-05-2015

Corresponding Author: Giorgia della Rocca

Dipartimento di Medicina

Veterinaria, Università Degli

Studi di Perugia, Italy

Email: giorgia.dellarocca@unipg.it

\section{Introduction}

All animals face dangers that can cause tissue damage and many of them carry out nociceptive responses that protect them from such damage.

Nociception is the essential event behind the concept of pain, since without it the experience of pain could not be realized. Nociception is indeed defined as the nervous process of detection and processing of a noxious stimulus. However, the term nociception is not synonymous with pain, the first being characterized by rapid involuntary reflex response that is not necessarily associated with a negative emotional response or feeling associated with pain. Actually pain is a "conscious awareness" associated with an emotional negative feeling, which originates from the perception of the actual or potential tissue damage (Elwood, 2011).

The ability of an animal to respond to a noxious stimulus only with an adaptive reflex response (nociception), or even through the conscious perception of the unpleasant experience (pain), involves a different concern about its welfare. It is therefore essential to determine which species are able to really feel pain and not only nociception, in order to adopt protective strategies when humans use such animals for several purposes.
Invertebrates are a broad group of animals that occupies more than $90 \%$ of the estimated 10 million species in the world (Kellert, 1993). They are largely used in scientific research, in nutrition and as attraction in aquariums and terrariums (Carere et al., 2011).

The use of invertebrates in scientific research is particularly frequent, because their breeding and reproductive characteristics make them very versatile and therefore useful for various types of studies and as biological models for other living beings, both animals and humans (Horvath et al., 2013). The experimental practices with invertebrates are extremely varied, including, among others, toxicological, electrophysiological and behavioral studies, not always exempt from causing even permanent damage to the animals. Nevertheless, the European current legislation is very weak with regard to the protection toward possible conditions of pain, suffering, distress or lasting harm that these animals may suffer as a result of the experimental procedure. Until a few years ago the use of animals for scientific research was regulated by the EU Directive 86/609/EEC, whose purpose was "the approximation of laws, regulations and administrative provisions of the Member States regarding the protection of animals used for experimental and other scientific purposes". However this Directive had several 
limitations, first of all it considered, among the animals to be protected against pain, stress and lasting harm, only vertebrate animal species. Invertebrate species, despite their wide use for experimental purposes, were not mentioned. In 2010, the new EU Directive 2010/63/EU was issued, with which new scenarios in the protection of animals used for experimental purposes are opened. In particular, this new Directive extends the range of cited animal species, including cephalopods. In fact, it says that "In addition to vertebrate animals including cyclostomes, cephalopods should also be included in the scope of this Directive, as there is scientific evidence of their ability to experience pain, suffering, distress and lasting harm.". For these species, it is therefore necessary to pay attention to the type of procedures to be applied and methods to be used, in order to safeguard their welfare.

Beyond the protection of invertebrates used for scientific research, concern about pain, suffering, distress and stress in these animals should be taken into account in other situations. In fact, many invertebrates are renowned for their products, such as food, pearls and shells (Horvath et al., 2013). With regard to cephalopods and decapod crustaceans, it is known that these animals are highly searched for culinary purposes. The commercial chain concerning these animals begins with fishing: this usually takes place by means of large nets that are left in the sea for a certain time before they are recovered; animals can be trapped in the nets for several days, suffering from discomfort and stress due to inability to obtain food or fights between trapped animals. Once hoisted on board, they are put in containers free of water and with ice; despite the fact that these animals are able to get oxygen even from the air, this capacity is extremely reduced as compared to that obtained in water, so they may suffer from dehydration and hypoxia until death. The chain ends in the kitchen: here the most unpleasant situations occur; in fact, decapod crustaceans and cephalopods are not always killed by "humane" methods: one of the best known examples is the practice of immersing live lobsters into boiling water. In this area laws intended to restrict or eliminate unpleasant situations in these animals are completely lacking, also because of a lack of awareness of operators and public concern about the possibility that these species can experience pain and suffering. This is most likely due to the lack of studies confirming the presence of neural circuits and electrophysiological processes involved in the perception of pain.

\section{Nociception or Pain?}

Although it is a fact that all animal species virtually possess the nerve circuits eliciting nociception, the investigation of the ability to feel pain in a given animal species is a very difficult process.

The Working Party of the Institute of Medical Ethics has identified seven criteria that, taken together, might provide evidence for pain experience in animals (Andrews et al., 2013). These criteria, that could be applied also to cephalopods, are the following (Andrews et al., 2013):

- "Possession of receptors sensitive to noxious stimuli, located in functionally useful positions on or in the body and connected by nervous pathways to the lower parts of the nervous system

- Possession of higher brain centers [in the sense of integration of brain processing], especially a structure analogous to the human cerebral cortex

- Possession of nervous pathways connecting the nociceptive system to the higher brain centers

- Receptors for opioid substances found in the central nervous system, especially the brain

- Analgesics modify the animal's response to stimuli that would be painful for a human

- An animal's response to stimuli that would be painful for a human is functionally similar to the human response (that is, the animal responds so as to avoid or minimize damage to its body)

- An animal's behavioral response persists and it shows an unwillingness to resubmit to a painful procedure; the animal can learn to associate apparently nonpainful with apparently painful events"

The presence of nociceptors has been demonstrated in most animal phyla. Nociceptors are present in anemones, annelids, molluscs, arachnids and in many other invertebrates, justifying their ability to respond to mechanical, chemical or thermal stimuli. Even the larvae of Drosophila are able to escape from the sting of a wasp (Elwood, 2011). In cephalopods, receptors that respond to noxious stimulation but which are not triggered by innocuous stimuli have been highlighted. Studies in Sepia officinalis showed the presence of receptors that are activated by mechanical and electrical stimuli, but do not respond to thermal stimuli; it was also observed that these receptors, as in vertebrates, undergo to short term (30 $\mathrm{min}$ ) and long term (24 h) sensitization (Crook et al., 2013). The nociceptive system seems thus to originate far behind in the evolutionary process. However, given the high taxonomic distribution of these receptors and their biological role, the demonstration of the sole presence of nociceptors is not sufficient to say that the species that possess them are able to perceive pain (Elwood, 2011). Further study that better investigate the distribution, activation thresholds, awareness and connections with the central nervous system that these receptors have in cephalopods are therefore advocated (Andrews et al., 2013).

The central nervous system of invertebrates is much less complex than that of a mammal. Since it was long thought that the cerebral cortex was necessary for the pain experience, the absence of such a structure in invertebrates has fostered the belief that for these species it is impossible to feel pain (Kim, 2014). However, it has 
been highlighted that some invertebrates are able to perform some complex functions while presenting a nervous structure morphologically different from that of mammals. It is therefore plausible that the same function is feasible by different animal species, despite the different morphological characteristics and this assumption can also be applied to the pain experience. Because many invertebrates possess a nervous structure somewhat complex, although different from that of vertebrates, it cannot be excluded that some of them have similar roles to those elicited by the cerebral cortex. In fact, the brain of some invertebrates is particularly complex, presenting a clear functional separation between different areas and this complexity could make plausible the experience of pain (Elwood, 2011). In particular, the nervous system of cephalopods is extremely developed. Albeit with a different morphology compared to vertebrates, the nervous system of these animals is structured in lobes hierarchically organized (Borrelli and Fiorito, 2008; Andrews et al., 2013). These lobes control the motor responses (including those of chromatophores), the blood system, the sensory activities, as well as learning and memory processes. With regard to the sensory activities, the nervous system of cephalopods is able to process a huge amount of sensory information transposed by millions of mechanical and chemical receptors placed on the arms' and suckers' skin. These animals are therefore equipped with complex central structures capable of performing functions similar to those performed by the vertebrate brain cortex. In addition, some areas of the brain of cephalopods are very similar in their organization to those of vertebrates: this is a further proof of closeness between the two classes (Andrews et al., 2013).

There are numerous studies describing the existence of paths that project the peripheral portions to the central nervous system of cephalopods, demonstrating the presence of connections between the peripheral receptors and the central nervous system. However it is not yet clear if specific pain pathways are among these projection pathways (Andrews et al., 2013). In describing the inputs that reach the vertical lobe, Wells (cited by Andrews et al., 2013) considered some of them as able to transmit pain. However, there is no evidence to support this theory, since it is not excluded that such a path is involved in the transmission of pleasure or other types of sensations different from pain. Considering the substantial involvement of the vertical and frontal lobes in the mechanisms underlying memory, which would probably take place by means of a process similar to the long-term potentiation occurring in the hippocampus of mammals, it would be very important to understand whether the information comes up from nociceptors to these lobes (Andrews et al., 2013).

The endogenous opioid system has maintained a high conservation during evolution. Therefore, phylogenetically it is likely that this system could be present and functional even in cephalopods. The presence of numerous neurotransmitters and neuropeptides (enkephalins and endorphins) that are involved in the painful response in vertebrates was highlighted in some invertebrates (flatworms, molluscs, annelids, crustaceans and insects). Enkephalin-like peptides are present in neurons of the palliovisceral lobe of Octopus vulgaris and receptors for methionine-enkephalin as well as delta opioid receptors have been found in the gastrointestinal tract, mantle and limbs of Octopus spp. However it should be remembered that in vertebrates the opioid system not only draws upon the responses of the animal to painful stimuli, but it has a very wide scope, from the regulation of metabolism to the endogenous control of certain growth hormones (e.g., modulation of steroids and of the endocannabinoid system). Therefore, the mere presence of opioid receptors and endogenous opioid-like substances is not sufficient to assert their antinociceptive role in invertebrates. The hypothesis that these substances have a function different from that expected and that in their place there are different molecules that take part in the painful response should be considered. Further studies must therefore be carried out to fully understand the actual role of the opioid system in cephalopods and other invertebrate species (Andrews et al., 2013).

A study on the effect of morphine on the defensive response of the crab Chasmagnathus granulatus showed a dose-dependent reduction of the crab's sensitivity to the nociceptive stimulus (electrical shock), but no inhibition occurred when the same dose was administered with naloxone, suggesting that morphine acts through an opiate receptor (Lozada et al., 1988). On the other end, it has been reported that morphine at least in crustaceans, produced a general effect of non-responsiveness rather than a specific analgesic effect (Barr and Elwood, 2011). There are no specific studies about the effect of analgesics in cephalopods. Actually, these species do not receive analgesics during surgical practices and post-operatively (Cooper, 2011). Studies on potential substances with analgesic action in cephalopods should be advocated. The evidence supporting the presence of opioid receptors should be enough to spur the investigation into the analgesic effect that these molecules have in cephalopods; however, special attention to the general inhibitory effect on behavioral reactions by non-selective opioids like morphine should be paid (Andrews et al., 2013).

With regard to behavioural responses to noxious stimuli that are functionally similar to those of humans and evidence of learning in relation to painful stimuli, there are many evidences that invertebrates could feel pain and not only apply nociceptive responses. Indeed, beyond the reflex responses of subtraction to painful stimuli, associated exclusively to nociception, the implementation of learned behaviors (that persist over time implying the existence of a pain memory) consistent with the experience of pain was also highlighted in invertebrates. Experiments conducted on insects, molluscs and crustaceans have shown that, after an electrical stimulation, the animals adopt avoidance strategies (e.g., taking refuge in a safe area) before new 
noxious stimulus is applied and this activity cannot be interpreted simply as a reflex response. Further experiments on shrimps showed how these animals seem to be aware of the specific location of a painful stimulus, directing their attention to the exact point of the body where the stimulus was applied (eg. prolonged rubbing with a claw in one eye which was instilled with a drop of acetic acid) (Elwood, 2011). A study of the response of Eledone cirrhosa against skin wounds showed how the animal tends to cover the wound site with a limb, or string it with the tip of another limb, showing a certain degree of attention to the injured site (Andrews et al., 2013). The complexity and duration of these responses are consistent with the idea of a central processing of the information and then pain, going well beyond the reflex subtraction response associated with nociception. Another element that allows to lean toward the possibility that also in invertebrates there is not only nociception but also pain is the ability of these animals to implement tradeoffs between avoidance reactions and other activities, making motivational choices related to contingent needs. Such choices involve some form of information processing, confirming the presence of sufficient cognitive skills that are well associated with the possibility of experiencing pain. In a study on crabs, it was noted that these animals were less likely to leave their shell, when subjected to an electrical stimulation, if they perceived the smell of a predator in the surrounding environment, suggesting the implementation of a compromise between pain and danger. The octopus provides another example of this compromise. They are skilled predators of crabs, but when crabs keep a stinging anemone between their claws, they drastically change the hunting technique, although this becomes much less effective for catching food (Elwood, 2011).

However, we must remain cautious in comparing behavioral responses to potentially painful stimuli realized by various animal species. For example, although studies using electric shocks and negative reinforcements in order to evaluate the learning and memory have proved that cephalopods are able to distinguish harmful stimuli from not harmful ones, it is not obvious that the response to such stimuli is necessarily due to the activation of nociceptors. It could in fact be due to the activation of other receptors and may also result in unpleasant feelings that are different when compared to the humans pain experience. The attention to the injured site (eg. resting the limb above the wound) is not necessarily indicative of a conscious perception of pain. In fact, many aquatic animals such as fish, amphibians and cephalopods, secrete from the body surface copious amounts of mucus enriched with an antimicrobial peptide. A cephalopod that puts in place a mechanism of protection of a wound could therefore be aware of the presence of the wound, but if this response is related to the presence of illness and pain or rather to disinfection purpose is not yet known. This underlines the importance of a good level of knowledge of the biology of cephalopods in order to understand their attitudes (Andrews et al., 2013).

\section{Diagnosis of Pain in Cephalopods: Physiological and Behavioral Responses?}

In vertebrates, physiological and behavioral indicators are investigated to assess the reaction to a painful stimulation. The same indicators have been taken into account to investigate the presence of pain in invertebrates.

\section{Physiological Indicators}

Physiological indicators that are taken into consideration for invertebrates are the same that are used to evaluate the conditions of suffering and stress in vertebrates. However, if in the latter these indicators represent a fairly reliable and minimally invasive method of monitoring, in cephalopods this is not always true. In cephalopods, the indicators usually monitored are cardiorespiratory functions, defecation and vomiting, feeding behavior, release of ink and some blood parameters. The measurement of the heart and respiratory rate requires rather invasive techniques, such as the implantation of venous catheters, or methods that imply the presence of operators near to the animal; these two factors represent stressful events for the animal, thus altering the results of the measurements. Other measurements, although more easily performed, do not lead to scientifically evaluable results, due to high inter-subjects variability, nonstandardized methods and lack of known ranges with which to compare the obtained results (Andrews et al., 2013).

\section{Behavioral Indicators}

On the other hand, behavioral indicators can be suitable for the evaluation of the sensory experiences of cephalopods. Obviously, in order to identify those behaviors that may express the presence of pain in a given animal species, it is essential to know the behavioral repertoire or body pattern of the concerned species.

With reference to cephalopods, a "body pattern" is the product of a potential change of shape and color of the body, caused by combinations of color, structural, postural and locomotor components. The patterns expressed by cephalopods and their association with the information communicated by each of them turn out to be very complex, as many messages are transmitted through a combination of multiple overlapped patterns.

The collection of studies done over the years has led to the creation of a catalog of body pattern of cephalopods (Borrelli et al., 2006), which collects the entire behavioral repertoire that these animals can express through their body. From such a quantity and variety of behaviors showed by these animals, it might be possible to identify those behaviors that more than others could be associated with the presence of stress, suffering and pain and to conceive a method of objective assessment. 
Table 1. Components and body patterns likely associated to stimuli that generate stress, distress, suffering or pain in Octopus vulgaris (Borrelli et al., 2006)

\begin{tabular}{|c|c|}
\hline Chromatic light components & Expanded suckers \\
\hline & Eye flash \\
\hline & Lateral white stripes \\
\hline & Median white stripes \\
\hline Chromatic dark components & Arm bars \\
\hline & Branchial hearts \\
\hline & Eye ring(s) \\
\hline Textural components & Rough skin \\
\hline Postural components & Arms raised \\
\hline & Arms splayed \\
\hline & Arms spread \\
\hline & Arms twisted \\
\hline & Arms upturned \\
\hline & Curved forearms \\
\hline & Funnel directed towards external stimulus \\
\hline Locomotor components & Arms slap \\
\hline & Displacement activity \\
\hline & Full withdrawal response \\
\hline & Withdrawal manoeuvre \\
\hline Body pattern and Displays & Acute Resemblance \\
\hline & Aggressive Flush \\
\hline & Broad Conflict Mottle \\
\hline & Camouflage \\
\hline & Curled Arm Swimming \\
\hline & Dymantic \\
\hline & Flamboyant \\
\hline & Passing Cloud \\
\hline & Striped phases \\
\hline & Sucker display \\
\hline & Triple response \\
\hline Feeding behavior & Conflict posture \\
\hline
\end{tabular}

Table 2. Medications utilized for invertebrate pain (Table adapted from Lafferty et al., 2014, with permission)

\begin{tabular}{|c|c|c|}
\hline Agent & Dose & Comment \\
\hline \multirow[t]{2}{*}{ Ketamine } & $0.025-0.1 \mathrm{mg} / \mathrm{kg}$ & Anesthesia within $15-45 \mathrm{sec}$ \\
\hline & $90 \mu \mathrm{g} / \mathrm{g}$ IM & Duration $\leq 1 \mathrm{~h}$ \\
\hline Lidocaine & $30 \mu \mathrm{g} / \mathrm{g}$ IM & Injected intrathoracically; duration $25 \mathrm{~min}$ \\
\hline Procaine & $25 \mathrm{mg} / \mathrm{kg}$ & Anesthesia within $20-30 \mathrm{sec}$, duration $2-3 \mathrm{~h}$ \\
\hline \multirow[t]{2}{*}{ Xylazine $\mathrm{HCl}$} & $70 \mathrm{mg} / \mathrm{kg}$ & Anesthesia within 5-6 min, duration $45 \mathrm{~min}$ \\
\hline & $16-22 \mathrm{mg} / \mathrm{kg}$ & Anesthesia within 2-3 min \\
\hline \multirow[t]{2}{*}{ Ethanol } & $3 \%(30.0 \mathrm{~mL} / \mathrm{L})$ & Induction \\
\hline & $1.5 \%(15 \mathrm{~mL} / \mathrm{L})$ ethanol in seawater & Anesthetic maintenance \\
\hline Isoflurane & $5-10 \%$ & Anesthesia of terrestrial invertebrates \\
\hline Sevoflurane & $5-10 \%$ & Anesthesia of terrestrial invertebrates \\
\hline $\mathrm{CO}_{2}$ & $10-20 \%$ & Anesthesia of terrestrial invertebrates \\
\hline $\begin{array}{l}\text { MS-222 } \\
\text { is }\end{array}$ & $100 \mathrm{mg} / 1 \mathrm{~L}$ of water & $\begin{array}{l}\text { Buffering MS- } 222 \text { with the addition of sodium bicarbonate } \\
\text { recommended for use in invertebrates with sensitive skin, } \\
\text { such as snails and slugs. }\end{array}$ \\
\hline \multirow[t]{2}{*}{ MS-222 and benzocaine } & $0.4 \mathrm{~g} / \mathrm{L}$ & In seawater for both chemicals \\
\hline & $0.4 \mathrm{~g} / \mathrm{L}$ & \\
\hline Gramine & $0.01 \mathrm{mg} / \mathrm{mL}$ & Serotonin antagonist inhibits pumping \\
\hline Imipramine & $20 \mu \mathrm{g} / \mathrm{mL}$ & Stimulates pumping; high concentration act as anesthetic \\
\hline Ivermectin & $0.05 \mathrm{ng} / \mathrm{mL}$ & Inhibits pumping \\
\hline Muscimol & $2 \mu \mathrm{g} / \mathrm{mL}$ & This GABA agonist inhibits pumping \\
\hline Serotonin & $1 \mathrm{mg} / \mathrm{mL}$ & Stimulates pumping \\
\hline
\end{tabular}

With specific reference to the Octopus vulgaris, among the countless body pattern described in the catalog it would be possible to select those which could be associated with stimuli that generate stress, distress, suffering or pain (Table 1).

The attitudes most significant in this regard could include:

\section{Camouflage}

It is a behavioral state adopted by all cephalopods as a primary defense against predators. The camouflage includes many different patterns and produces an effect of similarity between the body and the surrounding environment. This feature can be achieved by spots, dots or flecks of color on 
the body, dark color of the eyes, indefinite contours of the body, flattening of the head in case of aggression in an open space, swelling of the body when attached to rocks or seaweed and by means of materials found on the seabed and kept with the suction cups on the body surface.

\section{Dymantic}

It is an aggressive and hostile pattern facing a likely predator or other alarming stimuli. The term "dymantic" or alarmed was originally created to describe the black spots on the thighs expressed by many frogs when they jump to divert the attention of an aggressor.

\section{Passing Clouds}

It is perhaps the most extraordinary effect manifested by cephalopods. It is due to a dark reddening that moves very fast along the body, from over the head to the rear of the body. A second cloud of color can start when the first is still not finished, creating an effect similar to the passage of waves on the body of the animal. This attitude runs in both intra- and extra-specific contexts: is usually manifested against other cephalopods or toward predators or humans.

\section{Triple Response}

It is the sequence of actions put in place by Octopus in response to alarming stimuli, such as predators or intruders. The first reaction is to disappear, showing a camouflage pattern, which is maintained until when the disturbing element go away or approach too much. If this situation occurs, the Octopus passes to the second stage: reappears and creates an alarmed pattern (dymantic), trying to upset and confuse the predator. Finally, the last reaction is to divert the attention of the intruder and is implemented only a few moments before he realizes the attack. The Octopus escapes emitting a strong jet of water in the direction of the attacker and simultaneously releasing a cloud of ink. Consequently, the attack usually occurs toward the load of black cloud and not the prey. This triple response consists of an elaborate sequence of subterfuge: A series of wrong information through various mechanisms, such as hide, confusing or shift attention.

\section{Withdrawal Maneuver}

When an Octopus is disturbed, his first reaction is to withdraw and bring the arts on the head and body to protect itself, leaving the suckers exposed. When the noxious stimulus is particularly strong and persistent, the animal can perform the subtraction maneuver.

\section{Eye Rings}

The animal shows a particular coloration around the eyes in case of trouble. It can be expressed, albeit only fleetingly and unilateral, in the presence of moderate stimuli, such as the arrival of a fish, becoming more pronounced in case of alarming input (Borrelli et al., 2006).
Obviously, further studies are needed in order to allow an unequivocally association between a given behavior and the presence of pain.

\section{Analgesia and Anesthesia for Invertebrates}

There is currently little or no information available pertaining to analgesia for invertebrates. However, the use of general anesthesia is suggested as a provisional practice whenever possible (Cooper, 2011; Lewbard and Mosley, 2012; Zachariah, 2012).

With particular reference to cephalopods, anesthetics and analgesic should be administered in the following situations: to practice both minor and major surgical procedures, in order to increase the ease of handling and restraint of the animal and to limit the perception of pain; for implantation of catheters or recording devices; to facilitate handling within specific assessments, such as ultrasound measurement of the size of the brain; for sampling body fluids or tissues by means of biopsies; to simplify the administration of drugs through the branchial heart; for the transport; to solve veterinary problems (Andrews et al., 2013).

The molecules utilized for invertebrate pain are showed in Table 2 (Lafferty et al., 2014).

In cephalopods, anesthetic agents that are usually used in the experimental field are the same that are used for other aquatic species. Magnesium hydrochloride, clove oil, Carbon Dioxide (CO2), ethanol, hypothermia are among the most used compounds. Although quite numerous in terms of molecules, the knowledge about their actual effectiveness, potency and side effects are still rather scarce (Andrews et al., 2013).

With regard to analgesics, very little is known about the effect of various analgesics in these animals. Gunkel and Lewbart (2008) suggest the use of ketoprofen and butorphanol during the entire duration of the procedure; however, the proposed doses are taken from previous studies performed on fish and amphibians.

With the introduction of the Directive 2010/63/EU the use of anesthesia and analgesia has became mandatory during scientific research, except for those occasions when this would be more traumatic than the procedure itself and/or incompatible with the purpose of the project.

The need to apply the new legislation will hopefully encourage numerous studies to define appropriate anesthetic and analgesic treatments.

\section{Conclusion}

Despite invertebrates are widely used in many different fields, rules for respecting their welfare are often lacking, especially when compared to vertebrates (Elwood et al., 2009). However, with the new scientific discoveries on the nervous system of cephalopods and decapod crustaceans, researchers are realizing the urgency to protect these animals from suffering and mistreatment (Horvath et al., 2013). 
The above, in fact, shows that despite some progress has been made especially at the legislative level, further studies are advocated to ensure to invertebrate species treatments comparable to that given to vertebrates.

Scientific studies conducted so far in order to investigate the ability of pain perception in some species of invertebrates, particularly cephalopods, have not yet confirmed this feature. However, more and more elements support the hypothesis that this species is able to perceive noxious stimuli as painful. Indeed:

- There is now an accurate conception of what is their nervous system and how complex it is

- Receptors similar to those that in vertebrates act as nociceptors have been evidenced

- The ability to learn what actions can be played and which not following a unpleasant experience has been demonstrated

- The full behavioral repertoire of cephalopods has been analyzed and many attitudes likely applied in response to adverse noxious or stressful stimuli have been highlighted

However, scientific research must continue and further studies should be planned and actuated to better identify neurophysiological paths and biochemical mechanisms involved in the transduction, transmission, modulation and integration of painful stimuli and to deepen knowledge in areas such as the recognition and management of pain, anesthesia, methods for euthanasia and other related still poorly investigated areas.

\section{Author's Contributions}

Giorgia della Rocca: Conceived the review and wrote the manuscript.

Alessandra Di Salvo: Searched for references.

Giacomo Giannettoni: Contributed to write the text.

Mary Ellen Goldberg: Contributed to the contents and checked for English spelling.

\section{Ethics}

No ethical issues are concerned with the present article.

\section{References}

Andrews, P.L.R., A.S. Darmaillacq, N. Dennison, I.G. Gleadall and P. Hawkins et al., 2013. The identification and management of pain, suffering and distress in cephalopods, including an aesthesia, analgesia and humane killing. J. Experimental Marine Biology Ecology, 447: 46-64.

DOI: $10.1016 /$ j.jembe.2013.02.010
Borrelli, L., F. Gherardi and G. Fiorito, 2006. A Catalogue of Body Patterning in Cephalopoda. 1st Edn., Firenze University Press, ISBN-10: 88-8453-376-7, pp: 626.

Borrelli, L. and G. Fiorito, 2008. Behavioral Analysis of Learning and Memory in Cephalopods. In: Learning and Memory: A Comprehensive Reference, Byrne J.J. (Ed.), Academic Press Oxford, ISBN-10: 978-0-12-370509-9, pp: 605-627.

Barr, S. and R.W. Elwood, 2011. No evidence of morphine analgesia to noxious shock in the shore crab, Carcinus Maenas. Behavioural Processes, 86: 340-344. DOI: 10.1016/j.beproc.2011.02.002

Carere, C., J.B. Wood and J. Mather, 2011. Species differences in captivity: where are the invertebrates? Trends Ecol. Evol., 26: 211. PMID: 21316791

Council Directive of 24 November 1986 on the approximation of laws, regulations and administrative provisions of the Member States regarding the protection of animals used for experimental and other scientific purposes (86/609/EEC).

Cooper, J.E., 2011. Anesthesia, analgesia and euthanasia of invertebrates. ILAR J., 52: 196-204. DOI: 10.1093/ilar.52.2.196

Crook, R.J. and E.T. Walters, 2011. Nociceptive behavior and physiology of molluscs: Animal welfare implications. ILAR J., 52: 185-195. DOI: 10.1093/ilar.52.2.185

Directive 2010/63/EU of the european parliament and of the council of 22 september 2010 on the protection of animals used for scientific purposes.

Elwood, R.W., 2011. Pain and Suffering in Invertebrates? ILAR J., 52: 175-182. DOI: 10.1093/ilar.52.2.175

Elwood, R.W., S. Barr and L. Patterson, 2009. Pain and stress in crustaceans? Applied Anim. Behav. Sci., 118: $128-136$ DOI: $10.1016 /$ j.applanim.2009.02.018

Gunkel, C. and Lewbart G.A., 2008. Anesthesia and analgesia of invertebrates. In: Anesthesia and Analgesia in Laboratory Animals. Fish, R., P.J. Danneman, M. Brown and A. Karas (Eds.), Academic Press, San Diego, ISBN-10: 0080559832, pp: 535-545.

Horvath, K., D. Angeletti, G. Nascetti and C. Carere, 2013. Invertebrate welfare: an overlooked issue. Ann. Ist. Super Sanita, 49: 9-17. PMID: 23535125

Kellert, S.R., 1993. Values and Perceptions of invertebrates. Conservation Biology, 7: 845-855. DOI: $10.1046 /$ j.1523-1739.1993.740845.x

Kim, A.B., 2014. No brain, no pain? The Triple Helix .

Lafferty, K., S.J. Cital and M.E. Goldberg, 2014. Analgesia in Exotic Animals. In: Pain Management for Veterinary Technicians and Nurses. Goldberg, M.E. (Ed.), Wiley-Blackwell, Ames, IA, ISBN-10: 111855552X, pp: 216-262. 
Lewbard, G.A. and C. Mosley, 2012. Clinical anesthesia and analgesia in invertebrates. J. Exotic Pet Medicine, 21: 59-70.

DOI: $10.1053 /$ j.jepm.2011.11.007

Lozada, M., A. Romano and H. Maldonado, 1988. Effect of morphine and naloxone on a defensive response of the crab Chasmagnathus granulatus. Pharmacol Biochem Behav., 30: 635-640.

DOI: $10.1016 / 0091-3057(88) 90076-7$
Zachariah, T.T., 2012. Invertebrate Animal Welfare. In: Invertebrate Medicine. G.A. Lewbart (Ed.), WileyBlackwell, Ames, IA,

ISBN-10: 0813817587, pp: 445-449. 